\title{
A Smart Shoe Insole to Monitor Frail Older Adults' Walking Speed: Results of Two Evaluation Phases Completed in a Living Lab and Through a 12-Week Pilot Study
}

Antoine Piau ${ }^{1,2,3}$, MD, PhD; Zara Steinmeyer ${ }^{1}, \mathrm{MD}$; Yoann Charlon ${ }^{2}, \mathrm{PhD}$; Laetitia Courbet ${ }^{4}$, PhD; Vincent Rialle ${ }^{4}$, $\mathrm{PhD}$; Benoit Lepage ${ }^{5}$, PhD; Eric Campo ${ }^{2}$, PhD; Fati Nourhashemi ${ }^{1,3}, \mathrm{MD}, \mathrm{PhD}$

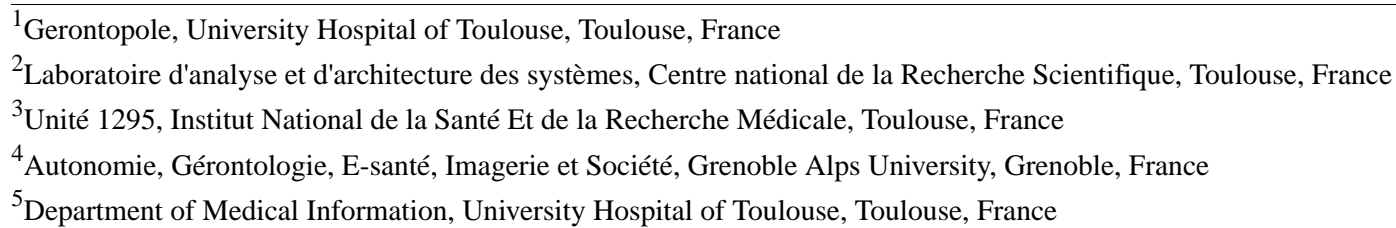

\section{Corresponding Author:}

Antoine Piau, MD, PhD

Gerontopole

University Hospital of Toulouse

24 rue du pont St Pierre

Toulouse, 31400

France

Phone: 33561323010

Fax: 33561325265

Email: antoinepiau@hotmail.com

\section{Abstract}

Background: Recent World Health Organization reports propose wearable devices to collect information on activity and walking speed as innovative health indicators. However, mainstream consumer-grade tracking devices and smartphone apps are often inaccurate and require long-term acceptability assessment.

Objective: Our aim is to assess the user acceptability of an instrumented shoe insole in frail older adults. This device monitors participants' walking speed and differentiates active walking from shuffling after step length calibration.

Methods: A multiphase evaluation has been designed: 9 older adults were evaluated in a living lab for a day, 3 older adults were evaluated at home for a month, and a prospective randomized trial included 35 older adults at home for 3 months. A qualitative research design using face-to-face and phone semistructured interviews was performed. Our hypothesis was that this shoe insole was acceptable in monitoring long-term outdoor and indoor walking. The primary outcome was participants' acceptability, measured by a qualitative questionnaire and average time of insole wearing per day. The secondary outcome described physical frailty evolution in both groups.

Results: Living lab results confirmed the importance of a multiphase design study with participant involvement. Participants proposed insole modifications. Overall acceptability had mixed results: low scores for reliability (2.1 out of 6) and high scores for usability (4.3 out of 6) outcomes. The calibration phase raised no particular concern. During the field test, a majority of participants (mean age 79 years) were very (10/16) or quite satisfied (3/16) with the insole's comfort at the end of the follow-up. Participant insole acceptability evolved as follows: 63\% (12/19) at 1 month, 50\% (9/18) at 2 months, and 75\% (12/16) at 3 months. A total of 9 participants in the intervention group discontinued the intervention because of technical issues. All participants equipped for more than a week reported wearing the insole every day at 1 month, 83\% (15/18) at 2 months, and 94\% (15/16) at 3 months for 5.8, 6.3, and 5.1 hours per day, respectively. Insole data confirmed that participants effectively wore the insole without significant decline during follow-up for an average of 13.5 days per 4 months and 5.6 hours per day. For secondary end points, the change in frailty parameters or quality of life did not differ for those randomly assigned to the intervention group compared to usual care.

Conclusions: Our study reports acceptability data on an instrumented insole in indoor and outdoor walking with remote monitoring in frail older adults under real-life conditions. To date, there is limited data in this population set. This thin 
instrumentation, including a flexible battery, was a technical challenge and seems to provide an acceptable solution over time that is valued by participants. However, users still raised certain acceptability issues. Given the growing interest in wearable health care devices, these results will be useful for future developments.

Trial Registration: ClinicalTrials.gov NCT02316600; https://clinicaltrials.gov/ct2/show/NCT02316600

(JMIR Mhealth Uhealth 2021;9(7):e15641) doi: 10.2196/15641

\section{KEYWORDS}

frail older adults; walking speed; outpatient monitoring; activity tracker; shoe insert

\section{Introduction}

Frailty is an age-related syndrome characterized by a decline in biological reserves with an increased risk of impaired autonomy and death [1]. As such, implementing intervention programs promoting physical activity is essential in preventing functional decline [2-4]. To measure the efficacy of these programs, it seems necessary to monitor frailty indicators and adherence over time [5,6]. Frailty was defined according to the five Fried criteria (slow gait speed, low physical activity, unintentional weight loss, exhaustion, and muscle weakness) [1]. Participants with a score of 0 were robust, 1 to 2 were considered prefrail, and 3 to 5 frail.

Among these criteria, consistent data indicates that walking speed is one of the strongest to predict adverse outcomes $[7,8]$. Currently, a patient walking speed is evaluated during clinical consultations by manually measuring the time the patient takes to walk 15 feet. This discrete assessment often fails to detect changes in day-to-day walking speeds and does not reflect walking speeds in everyday environments. As such, continuous ambulatory monitoring would ensure precise monitoring of a patient's health and better support medical diagnosis, especially by capturing a patient's decrease in physical activity and walking speed profile [9].

Currently, physical activity assessments are often based on self-reported questionnaires with poor or inconsistent reliability $[10,11]$, highlighting the importance of objective measures. The World Health Organization's reports on aging and health propose the use of wearable devices to collect information on physical activity and gait speed as health indicators [5]. Digital technologies allow the monitoring of patient's physiological data in their environment and thus tracking of subtle changes over time [9,12-14]. For example, accelerometers provide an objective measure of physical activity over a few days compared to standard physical performance measures [15]. Moreover, physical activity feedback with wearable sensors may also be incentive to increase daily activity [16-19].

Several sensor-based tools have been proposed to assess frailty and walking speed. However, they do not allow monitoring walking speed and activity in real-life conditions over long periods of time both indoors and outdoors [20,21]. To date, walking analysis research in patients who are frail is limited, and most studies involve electronic walkways, camera systems, or force plates, which limits real-life monitoring [9,22].

Thus, assessing a device specifically designed to monitor this population is relevant. Currently, multiple consumer-grade monitoring devices are commercialized, such as wrist-worn fitness trackers or smartphone apps. However, their accuracy is under debate especially in monitoring gait speed in older populations [19,23-25]. As such, their results must be interpreted cautiously. A majority of these devices evaluate activity by a built-in sensor counting one's steps and thus does not help characterize a patient's type of walk from normal walking to shuffling.

Our hypothesis is that a shoe insole is acceptable for long-term monitoring of indoors and outdoors walking speed in real-life conditions. A wireless insole is discrete and does not stigmatize the patient, users do not have to remember to wear it every day because it is placed in the patient's walking shoes, and studies have shown that inertial feet sensors can accurately measure walking speed [25-27]. However, acceptability beyond a few hours of testing is not yet reported. The objectives of our multiphase study is to assess the technical feasibility (eg, wireless transmission and calibration protocol) and the acceptability from the user's perspective. The secondary objective is to describe the evolution of a patient's frailty syndrome and functional autonomy in both groups, quality of life, and health costs.

\section{Methods}

\section{Study Design and Setting}

In line with codevelopment and health technology assessment recommendations [28-30], we designed a two-phase pilot study [31] involving community-dwelling prefrail and frail older participants remotely followed at home. It consisted of, first, a noncomparative trial in a living lab and a 12-week prospective, parallel, randomized controlled clinical trial (field trial). The first trial lasted from September 2015 to January 2016. The second lasted from October 2016 to January 2019.

The living lab experiment was set up in a living lab at the University Institute of Technology in Blagnac, France (Maison Intelligente de Blagnac) located on the university campus [26]. This flat of $70 \mathrm{~m}^{2}$ is equipped with a networking infrastructure accessible to valid, frail, or disabled persons. It enables testing of technological devices in an environment similar to one's home setting but with controlled technical conditions. The living lab phase was carried out in two subphases: (1) 9 older adults were evaluated in the Maison Intelligente de Blagnac living lab and (2) 3 older adults among the 9 participants of the living lab were followed up with at home for a month.

During the first phase, participants were invited to complete a single session standard scenario in the Maison Intelligente de Blagnac living lab (45-minute sessions consisting of a walking 
tour in the living lab and outside, as per usual) after calibrating the insole to their own step length and after a 10-minute presentation of the product's objectives. During the second phase, volunteers were asked to use the device over a 1-month time period in their own homes (Figure 1). Participants were invited to wear the insole and use a touchpad without any additional instructions or training.

Figure 1. Description of the evaluation phases.

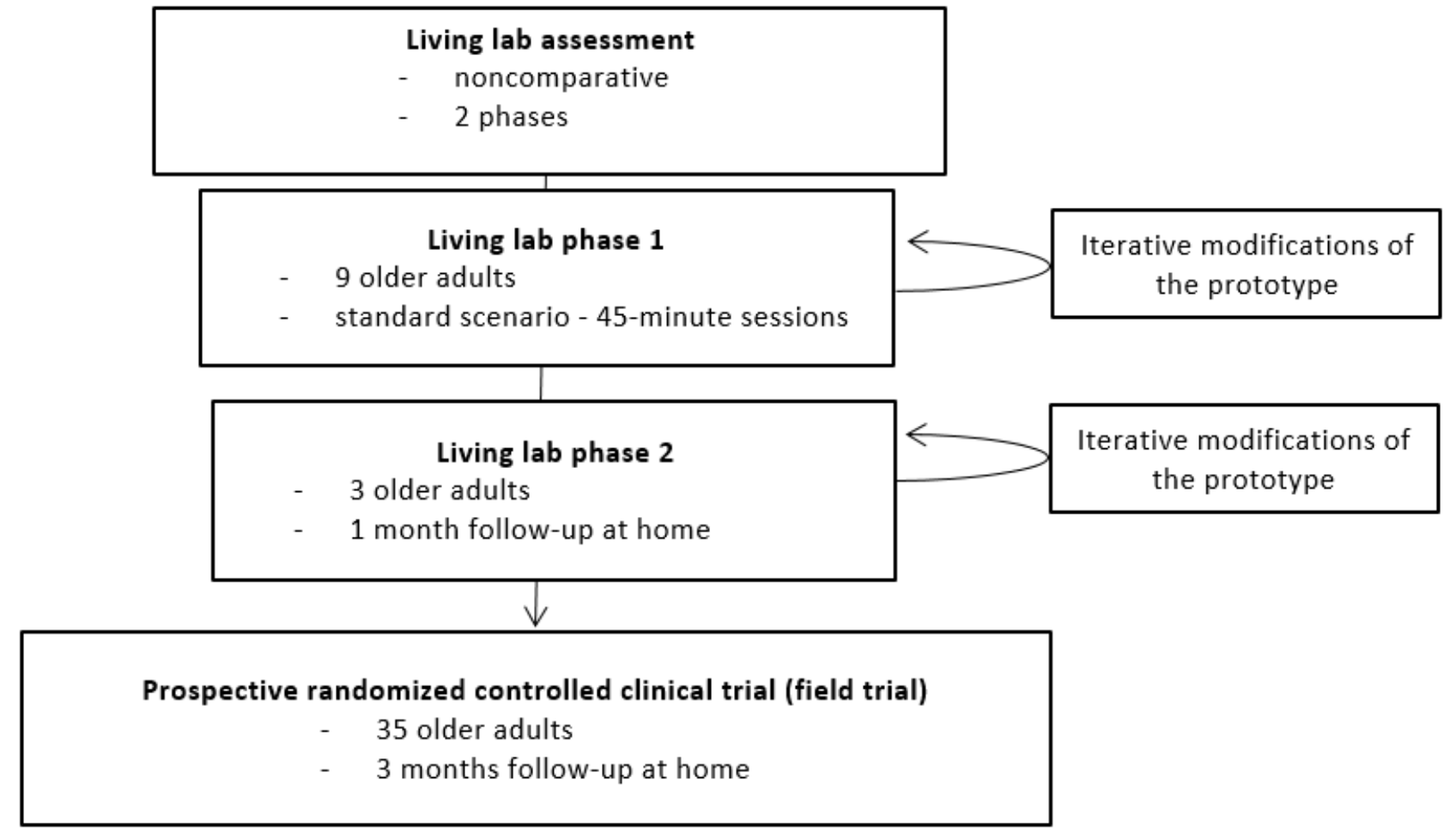

For the field trial, 35 participants were randomized following a 2:1 allocation into two distinct arms. Randomization was conducted independent of recruitment by the hospital's epidemiology department. The aim of randomization was to describe the secondary objectives for future effect size calculation. Participants were randomized in either the intervention (smart insole follow-up) or control group (standard follow-up) and enrolled for 12 weeks.

\section{Intervention Description}

Participants in the living lab phase and those randomized in the intervention group in the field trial phase were equipped with the instrumented shoe insole and were given a touchpad feedback app. The technological devices tested were a pair of insoles (only one insole is instrumented), a touch pad to collect data from the insole and inform the users about their activity (Bluetooth communication), and an induction charger to charge the shoe every night (Figure 2). The insole thickness is less than
2.5 millimeters at its thickest point ( $\operatorname{arch})$. The insole measures according to time (day, week, and month) the number of steps and walking distance, the average walking speed during walking periods, and active walking duration (as opposed to shuffling). Active walking was defined as continuous walking for at least 5 minutes with a tolerance of 1 minute (see Figure 3) considering that health benefits of aerobic exercise begin with any increase above the lowest levels of activity [32]. Moreover, the insole is calibrated on each participant's step length for an accurate measurement of walking speed, unlike consumer-grade devices. The walking speed measure algorithms and the lab test results of the insole have been previously described [26].

Both groups benefitted from a frailty assessment at a geriatric day hospital for frailty (GHF) as per usual follow-up. Indeed, GHFs are developed nationwide in France following the French national authority recommendations and may accommodate up to five patients per day. Patients benefit from a follow-up phone call at 3 months and at 1 year by a nurse. 
Figure 2. Overall technological device description. It includes a pair of insoles, an induction charger fitting in the shoe, a touchpad to collect data from the insole and provide feedback to the user, a secure remote database, and a web application for the patient and the physician. The insole is $2.5 \mathrm{~mm}$ at its thickest point (arch); it has a buffer memory and a flexible battery for walking comfort. If the battery is not recharged, an alert is issued to the user. The touchpad is presented here with a diagram of average walking speed and a diagram reporting active walking minutes.

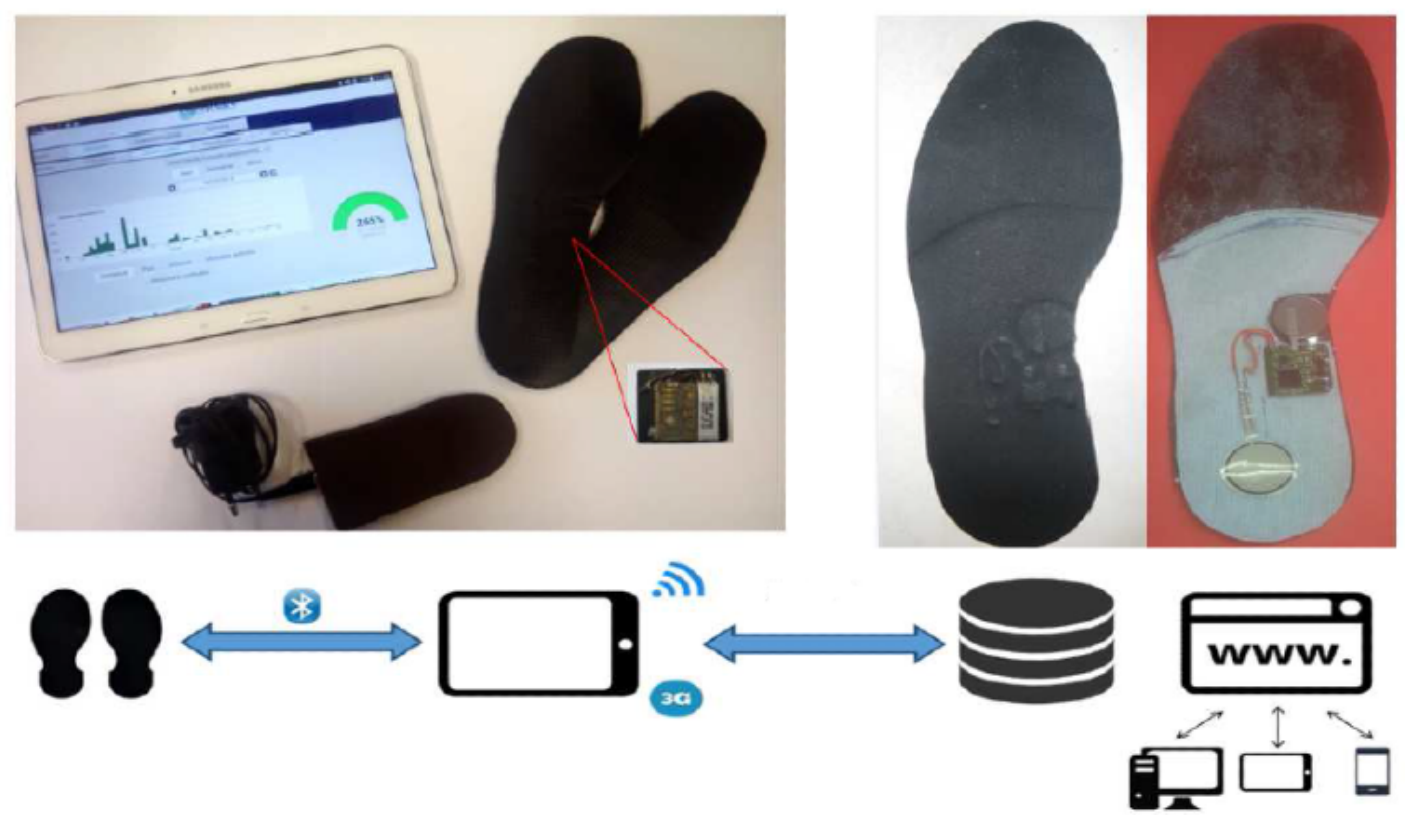

Figure 3. Active walking definition. The insole accounts for steps in any case.

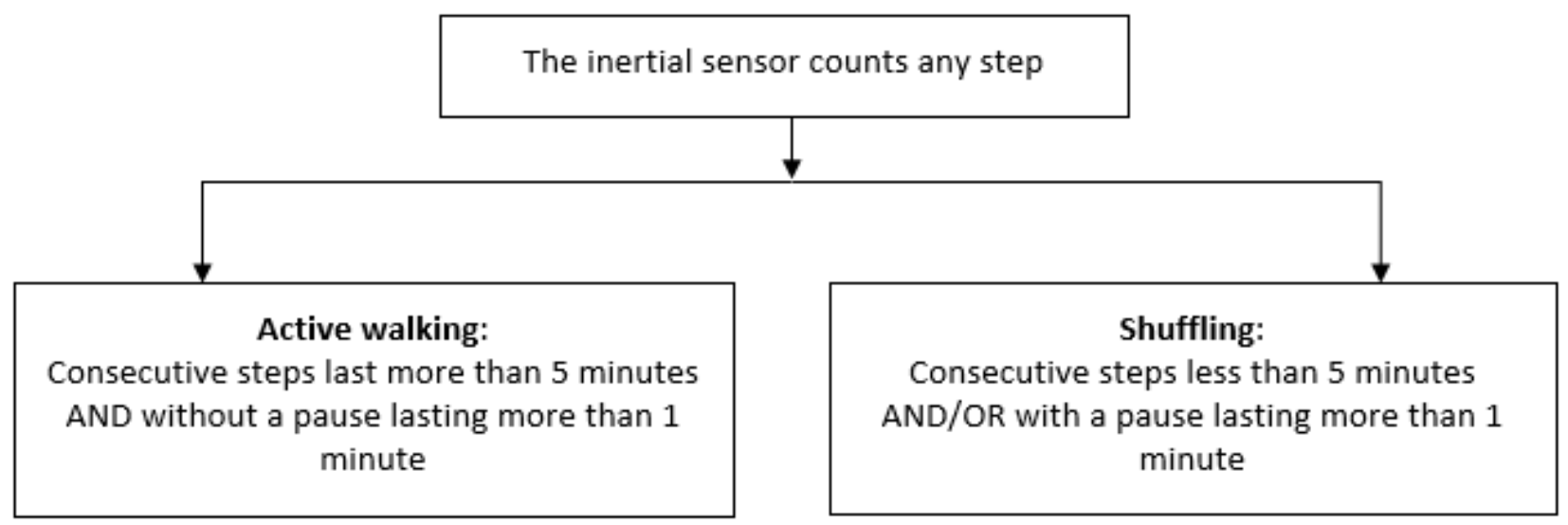

\section{Recruitment and Eligibility Criteria}

Study participants were recruited through the Toulouse University Hospital GHF (France). Ethical approval for the study was obtained from the regional independent ethics committee in September 2014 (ID-RCB: 2014-A00523-440). The trial is registered on ClinicalTrials.gov (NCT02316600). All participants provided written consent. Baseline assessments were conducted in-person at the GHF. The inclusion criteria were (both phases):

- Patients 65 years or older living independently at home

- Activity of Daily Living (ADL) score (ranges from 0 to 6; the higher the score, the higher the level of functional autonomy in daily life is; eg, walking or dressing) [33] of 4 or higher

- Mini-Mental State Examination (MMSE; ranges from 0 to 30; the higher the score, the higher the level of global cognition) [34] of 24 or higher
- Prefrail or frail according to Fried criteria [1], for the field trial only

The sole exclusion criteria was life expectancy of less than 12 months. We did not request any specific level of computer literacy, and none of the participants were familiar with digital tools. There was no financial compensation for participation.

\section{Data Collection Procedure}

At inclusion, sociodemographic data (gender, age, marital status, living place), frailty status [1], functional abilities with the ADL [33], and cognition with MMSE [34] were collected. For the field trial, other functional and physical scores were also assessed (Instrumental Activities of Daily Living [IADL] [35] and Short Physical Performance Battery [SPPB] scores [36], respectively).

In the living lab phase, semistructured interviews were conducted, focusing on technical feasibility and acceptability. We also scored the 3 home participants' satisfaction with the 
device at the end of the 1-month follow-up using the main themes identified during the interviews.

For the field trial, the primary outcome measured the acceptability of the device as follows:

- Qualitative indicators: acceptability assessment questionnaire based on the Quebec User Evaluation of Satisfaction With Assistive Technology scale [37] aimed to evaluate technology satisfaction degree from 1 to 5 (1: not satisfied at all; 5: very satisfied) and semistructured interview of the first 10 participants who completed follow-up.

- Quantitative indicators: number of days wearing the insole and average time of wearing the insole per day as declared by the participants and as measured by the insole and the number of connections to the app

The secondary outcome of the field trial measured:

- Frailty status according to the Fried criteria (slow gait speed, low physical activity, unintentional weight loss, exhaustion, and muscle weakness) [1]

- Lower extremity physical performance assessed with the SPPB (consisting of a balance test, a 3-meter gait test, and a 5 chair rises test; score ranging from 0 to $12 ; 12$ indicating the highest degree of functioning) [36]

- ADL [33]

- IADL (ranging from 0 to 8; the higher the score is, the better the participant's functional autonomy in daily life is; eg, driving) [35]

- Quality of life questionnaire (36-Item Short Form Health Survey [SF-36]; ranges between 0 and 100; greater score indicates better health-related quality of life) [38]

- EQ-5D-3L index, recording the patient's self-rated health in a five-digit health state profile, each comprised of three levels (the score is converted into a single summary number ranging from -0.59 to $1 ; 1$ indicating the highest level of perceived health) [39]

- Major medical events defined as any event leading to a hospitalization or an emergency admission

\section{- Health costs}

The semistructured interview was proposed to each participant at the end of the follow-up date (45 minutes for the living lab test, 1 month for the 3 living lab home testers, 3 months for the field test). The interview was face-to-face for the living lab group and by phone call for both home evaluations. Two researchers of the Age-Imaging-Modelization Laboratory (LC and VR; Joseph Fourier University Sociology Laboratory, Grenoble, France) conducted them. Each interview lasted 2 hours and was designed to explore key questions relating to acceptability. For the first phase of the study (living lab), nondirective exchanges were conducted to identify recurring themes. These themes guided the interviews of the field trial participants $(n=10)$. All interviews were transcribed. The transcripts were analyzed using a conventional content analysis along with a summative qualitative content analysis [40].

For the field phase, a clinical research assistant also contacted participants at 1, 2, and 3 months to evaluate secondary outcomes. For acceptability, the following question was systematically asked: "how well did you tolerate wearing the insole during the past month?" (5 possible answers were proposed: from totally tolerable to totally intolerable).

\section{Statistical Analysis}

For the field trial, we planned to include 35 participants complying with pilot study recommendations [41]. Qualitative variables were presented by effectives and percentages, quantitative variables by means and SDs. Likert-type items were handled as continuous variables.

Comparison tests were performed: quantitative variables with Wilcoxon-Mann-Whitney test and chi-square or Fisher tests for qualitative variables. Missing data was replaced with the mean values of the groups, allowing complete case analysis. A drawback of this approach is reduced variability and weakening of covariance and correlation estimates in the data.

The Department of Epidemiology of the Toulouse University Hospital in Toulouse conducted statistical analyses using Stata version 14.2 (Stata Corp).

\section{Results}

\section{Living Lab}

\section{Study Sample Characteristics}

A total of 9 participants were included, 6 (67\%) women and 3 men. The mean age was 70.1 (SD 2.3) years (range 65-75), and none of them presented any functional disability (Table 1).

Table 1. Characteristics of participants $(n=9)$.

\begin{tabular}{ll}
\hline Characteristics & Participants \\
\hline Gender (female), n (\%) & $6(67)$ \\
Age (years), mean (SD) & $70.1(2.3)$ \\
Activity of Daily Living score, mean (SD) & $5.9(0.3)$ \\
Mini-Mental State Evaluation score, mean (SD) & $29.6(0.5)$ \\
Frailty status (frail or prefrail), n (\%) & $1(11)$ \\
\hline
\end{tabular}

\section{Acceptability}

The research assistant of the study informed participants in the living lab in the following terms: "Walking every day is beneficial to your health (e.g., independence, cognition) and the insole is designed to quantify your activity and provide feedback that can help you progress and make you want to walk more." Interview feedback revealed participants' understanding 
of the device's objectives: 7 participants out of 9 cited prevent the risk of becoming dependent as a main objective, 5 physical activity follow-up, and 4 motivate us to be physically active. A total of 6 participants stated that wearing the insole could potentially encourage them to walk more. Indeed, real-time feedback would "motivate them to go out from home" and "stimulate their desire to adopt a healthier lifestyle." A total of 4 participants suggested that reminder texts would increase physical activity motivation if activity fell below objectives. There were 5 other participants that were confident in achieving the walking threshold without any help. One person reported that such a reminder would be intrusive. None of the participants expressed concerned with the calibration protocol. Most participants $(n=8)$ claimed that the insole was comfortable, light, and robust, and did not cause any discomfort. Nevertheless, flexibility and thickness were negatively highlighted by 2 of them with the fear of possible long-term use discomfort. Concerning the user touchpad interface, 5 participants expressed the need for a longer time practicing to assess acceptability. Several major themes were identified (example codes are given in parenthesis):

- Understanding and adhesion to the device's objectives (eg, facilitating role)

- Device acceptability (eg, comfort)

- Device usability (eg, reliability)

- Device adherence (eg, time wearing the insole)

The 3 home participants reported wearing the insole for 1 month without early dropout. According to the insole data, they wore the insole for 22,13 , and 13 days out of 30 , respectively. Total "active walking" time (as opposed to shuffling and therefore different from the number of steps) was 29 hours and 33 minutes
(7288 average steps per day), 4 hours and 11 minutes (average steps per day 1748), and 3 hours and 52 minutes (average steps per day 1574), respectively. Average walking speed (calculated from the participants average step length obtained during the calibration protocol and cadence measurement) was $0.90 \mathrm{~ms}^{-1}$, $0.75 \mathrm{~ms}^{-1}$, and $0.69 \mathrm{~ms}^{-1}$.

All participants claimed that the insole was comfortable and that "once they were placed in the subject's shoes, you tend to forget them." According to users, the most interesting feedback information was walking distance (more than number of steps, walking speed, or the active walking time). Some texts of the interface were considered too small and colors not appropriate by 1 participant with age-related visual impairment. Of the 3 participants, 2 declared that they would use this device if it was commercialized. No harms or unintended side effects were reported. Table 2 summarizes the 3 home participants' satisfaction with the device at the end of the 1-month follow-up. At the end of the interview, we asked them to rate their overall satisfaction with the device (score ranging from 0 "strongly disagree" to 6 "strongly agree"). The mean score was 3.1 (out of 6) for the facilitating role, 4.3 for user friendliness, and 2.1 for reliability outcomes.

Concerning technical feasibility, battery autonomy at home ranged from 30 to 48 hours, and no serious concern was raised about the induction charging system. Participants were satisfied with the daily routine, especially since there is no connection to be made for charging. One insole instrumentation was broken after 3 weeks of use. The 3 participants encountered synchronization problems between the insole and the touchpad (long latency). 
Table 2. Participants satisfaction with the device at the end of the 1-month follow-up.

\begin{tabular}{|c|c|}
\hline Participants satisfaction & Participant ratings ${ }^{\mathrm{a}}$, mean (individual) \\
\hline The device helps me to achieve my objectives (facilitating role) & $3.7(5,3,3)$ \\
\hline It motivates me to complete my activities (facilitating role) & $3.0(1,3,5)$ \\
\hline It helps me to be more efficient (facilitating role) & $3.0(5,2,2)$ \\
\hline The device is easy to install (user friendliness) & $2.7(0,5,3)$ \\
\hline The device is fun to use (user friendliness) & $5.0(5,5,5)$ \\
\hline Using it is effortless (user friendliness) & $5.3(5,5,6)$ \\
\hline I don't need written instructions (user friendliness) & $3.0(1,5,3)$ \\
\hline I easily learned to use it (user friendliness) & $4.7(5,5,4)$ \\
\hline I quickly became an expert in its use (user friendliness) & $4.7(6,4,4)$ \\
\hline It is easy to use (user friendliness) & $4.0(4,4,4)$ \\
\hline It is user-friendly (user friendliness) & $4.7(5,4,5)$ \\
\hline It is suitable for both frequent and infrequent users (user friendliness) & $5.0(5,5,5)$ \\
\hline I always remember how to use it (user friendliness) & $3.7(6,2,3)$ \\
\hline It is pleasant to use (user friendliness) & $3.3(5,2,3)$ \\
\hline I am always able to use the device (reliability) & $2.3(1,4,2)$ \\
\hline It always works as desired (reliability) & $2.7(3,1,4)$ \\
\hline It always does exactly what I want (reliability) & $1.3(0,2,2)$ \\
\hline It perfectly fits my needs & $2.0(1,0,5)$ \\
\hline I need to have one & $2.7(1,4,3)$ \\
\hline I will recommend it to a friend & $3.0(1,3,5)$ \\
\hline
\end{tabular}

${ }^{\mathrm{a}}$ Answers range from 0 to 6 : $0=$ strongly disagree, $1=$ disagree, $2=$ somewhat disagree, $3=$ no opinion, $4=$ somewhat agree, $5=$ agree, $6=$ strongly agree. The higher the rating, the better the satisfaction.

\section{End Users' Propositions}

Participants proposed changing the insole's design, the app, and the synchronization protocol. As a result, improvements were made before the field trial phase. To improve comfort and strength, the insole was modified by thermo-moulding, and the thickness of the electronics was reduced. The circuit was then varnished and encapsulated in epoxy glue and neoprene to protect it from impact and avoid friction. The participant's interface was also modified to improve text and graphics readability. Upon user request, a light encoder was added on the induction charger that changes from red to green when the charger is rightly positioned in the shoe. Finally, the communication protocol between the tablet and the insole was optimized, and a bug disrupting data transmission (synchronization after several days of use) was fixed.

\section{Field Trial}

\section{Study Sample Characteristics}

A total of 35 participants were included, 10 in the control group and 25 in the intervention group. In the intervention group, 6 participants left the study between visit one and visit two (3 because of defective equipment), 1 participant left the study between visit two and visit three because of defective equipment, and 2 participants left the study between visit three and visit four (1 for defective equipment).

The CONSORT (Consolidated Standards of Reporting Trials) flow diagram is presented in Figure 4 [42]. 
Figure 4. CONSORT (Consolidated Standards of Reporting Trials) flow diagram.

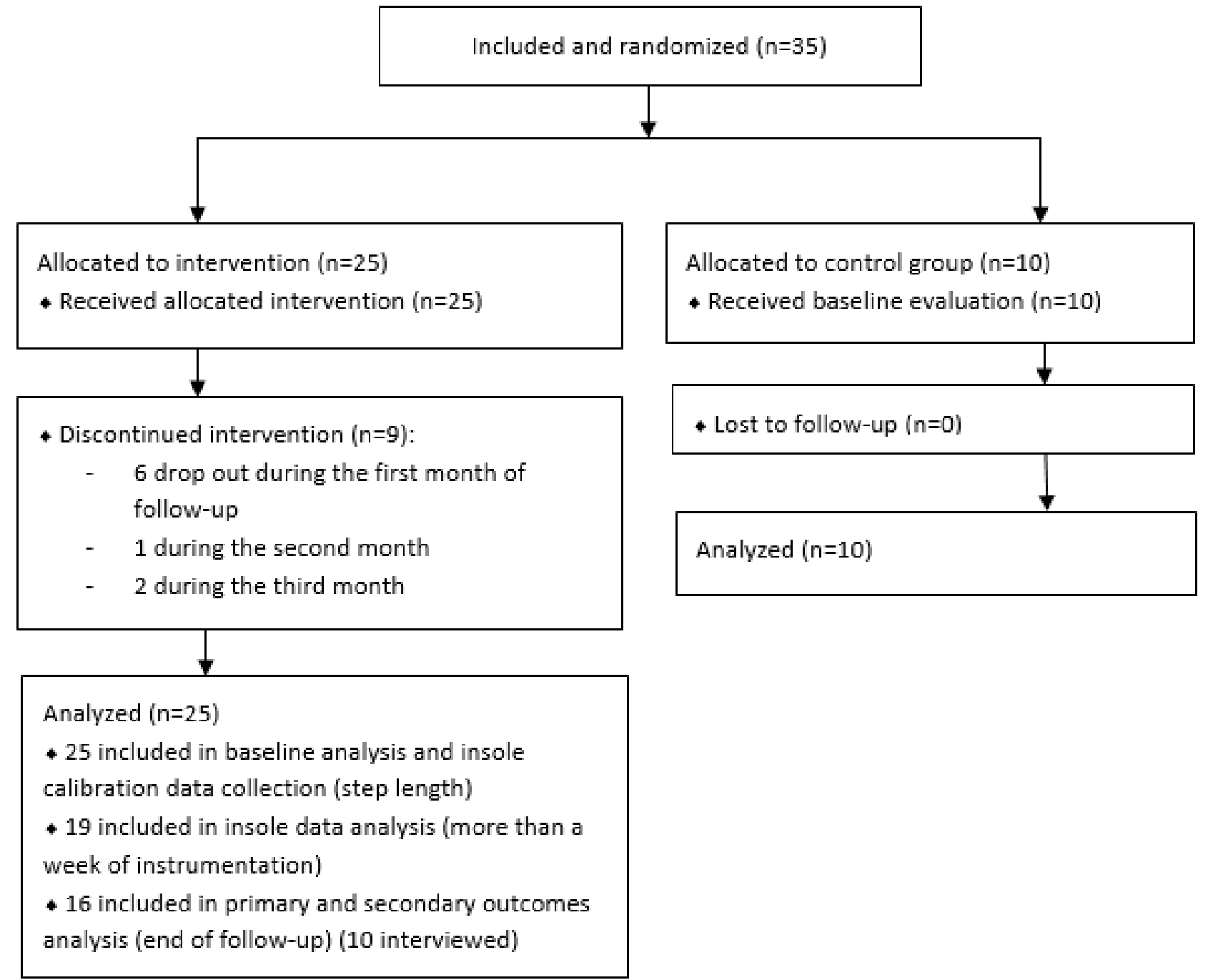

A total of $80 \%(8 / 10)$ of participants were women in the control group and $64 \%(16 / 25)$ in the intervention group. The average age was 79 (SD 5.8, range 70-89) years. One-third lived in collective housing and two-thirds in individual housing.
Participants were quite active; 72\% (18/25) reported walking every day in the intervention group compared to $70 \%(7 / 10$; $P=.92$ ) in the control one, and less than $10 \%$ reported not walking at all in both groups (Table 3 ). 
Table 3. Characteristics of participants ( $\mathrm{n}=35)$.

\begin{tabular}{|c|c|c|c|}
\hline Characteristics & Control group $(n=10)$ & Intervention group $(n=25)$ & $P$ value $^{\mathrm{a}}$ \\
\hline Age (years), mean (SD) & $77.8(5.9)$ & $79.3(5.9)$ & .05 \\
\hline Gender (female), n (\%) & $8(80)$ & $16(64)$ & .44 \\
\hline Education, $\mathbf{n}(\%)$ & & & .44 \\
\hline Low level & $1(10)$ & $0(0)$ & \\
\hline Middle level & $5(50)$ & $12(48)$ & \\
\hline High level & $4(40)$ & $13(52)$ & \\
\hline Marital status, n (\%) & & & .23 \\
\hline Married & $3(30)$ & $12(48)$ & \\
\hline Single, divorced, widower & $7(70)$ & $13(52)$ & \\
\hline Living arrangements, n (\%) & & & $>.99$ \\
\hline Alone ( $\mathrm{n}=9$ for control) & $6(67)$ & $15(60)$ & \\
\hline With other ( $\mathrm{n}=9$ for control) & $3(33)$ & $10(40)$ & \\
\hline $\mathrm{ADL}^{\mathrm{b}}$ score, mean (SD) & $4.8(1.5)$ & $5.0(1.4)$ & .79 \\
\hline IADL $^{\mathrm{c}}$ score, mean (SD) & $7.9(0.3)$ & $7.9(0.2)$ & .59 \\
\hline MMSE $^{\mathrm{d}}$ score, mean (SD) & $27.0(1.4)$ & $29.1(1.4)$ & .09 \\
\hline SPPB $^{\mathrm{e}}$ score, mean (SD) & $10.2(2.8)$ & $11.2(1.1)$ & .42 \\
\hline
\end{tabular}

${ }^{\mathrm{a}}$ Fisher test or Wilcoxon rank sum test.

${ }^{b}$ ADL: Activity of Daily Living.

cIADL: Instrumental Activities of Daily Living.

dMMSE: Mini-Mental State Evaluation.

${ }^{\mathrm{e}} \mathrm{SPPB}$ : Short Physical Performance Battery.

\section{Acceptability Results (Primary Outcome)}

\section{Qualitative Indicators}

Semistructured home interviews at the end of the 3-month follow-up $(n=10)$ reported that the insole was well tolerated. A total of $10(100 \%)$ participants declared wearing them every day. Most participants $(n=7,70 \%)$ affirmed that they did not need any incentive to wear the insole. Most claimed that the insole was comfortable, light, and robust, and did not cause any discomfort ( $\mathrm{n}=7,70 \% ; 3$ participants found them too thick). A total of 4 participants complained that the insoles did not fit in every type of shoe. The 3 participants who did not walk regularly (walk over short distances on a daily basis), declared that wearing the insole encouraged them to walk because "it stimulated their desire to surpass themselves" and "go out from home." Concerning the user interface on the touchpad, users

expressed some difficulties in handling the devices due to lack of habit (6 participants). As a result, they failed to read their data and did not understand its usefulness. These shortcomings led half of them (5/10) to almost abandon the tablet.

Regarding the tolerance of wearing the insoles during follow-up, $63 \%(12 / 19)$ of participants found it totally tolerable, $37 \%(7 / 19)$ quite tolerable at 1 month, 50\% (9/18) and 44\% (8/18) at 2 months, and finally increasing to $75 \%(12 / 16)$ and $19 \%(3 / 16)$ at 3 months. Concerning the acceptability questionnaire results at the end of the follow-up ( $n=16$; Table 4), the overall answer was "quite satisfied" or "very satisfied." The participants were "very satisfied" for weight; between "more or less satisfied" and "very satisfied" for dimensions, ease of adjusting, safety, robustness, and comfort; and less satisfied for ease of use and effectiveness in meeting their needs. 
Table 4. Questionnaire results in the intervention group at the end of the follow-up $(n=16)$.

\begin{tabular}{|c|c|c|c|c|c|}
\hline How satisfied are you with (...) your device ? & Not at all, n (\%) & Not very, n (\%) & More or less, $\mathrm{n}(\%)$ & Quite, n (\%) & Very, n (\%) \\
\hline ...the dimensions of... & $0(0)$ & $0(0)$ & $3(19)$ & $4(25)$ & $9(56)$ \\
\hline ...the weight of... & $0(0)$ & $0(0)$ & $2(12)$ & $0(0)$ & $14(88)$ \\
\hline ...the ease in adjusting... & $0(0)$ & $0(0)$ & $2(12)$ & $4(25)$ & $10(63)$ \\
\hline ...the safety of... & $0(0)$ & $0(0)$ & $4(25)$ & $2(12)$ & $10(63)$ \\
\hline ...the robustness... & $1(6)$ & $0(0)$ & $3(19)$ & $3(19)$ & $9(56)$ \\
\hline ...the ease of use... & $1(6)$ & $4(25)$ & $1(6)$ & $4(25)$ & $6(38)$ \\
\hline ...the comfort... & $0(0)$ & $1(6)$ & $2(12)$ & $3(19)$ & $10(63)$ \\
\hline ...the effectiveness... & $4(25)$ & $1(6)$ & $4(25)$ & $1(6)$ & $6(38)$ \\
\hline
\end{tabular}

\section{Quantitative Indicators}

A total of 25 participants were equipped with the insole, of which 6 for a duration of less than 7 days because of technical problems (Bluetooth communication with the touchpad) at the beginning of the study. Mean step length during calibration was 0.54 (SD 0.16) meters $(\mathrm{n}=25)$.

Apart from these 6 participants, $100 \%(n=19)$ of participants reported wearing the device every day at 1 month, $83 \%(15 / 18)$ at 2 months, and $94 \%(15 / 16)$ at 3 months of follow-up. Participants reported that the device was worn on average between 5.8 (SD 2.9), 6.3 (SD 6.4), and 5.1 (SD 3.7) hours per day at 1 month, 2 months, and 3 months, respectively. The mean number of days of wearing the insole according to the sensors data in a 3-month period was 29.2 (SD 28.7). If the participants with less than a week of instrumentation $(n=6)$ were excluded, the mean increased to 40.4 (SD 28.8) without significant decline during follow-up (14.2, 12.7, and 13.5, respectively). On average, participants wore the insole for 5.6 (SD 3.7) hours a day. These figures only take into account the days when the insole effectively transmitted data to the server, which excludes connection failure periods or days with insufficient battery charging. The participants connected to the web application on average 45.4 (SD 68.3) times during the follow-up, which corresponds to a mean number of 4.3 (SD 10.6) connections per day.

\section{Secondary Outcomes}

\section{Health Outcomes}

For health outcomes, there were no statistically significant differences between the two groups at baseline and at the end of follow-up. At baseline, there were $0 \%(0 / 10)$ frail and $70 \%$ (7/10) prefrail in the control group, compared to $0 \%(0 / 10)$ and $83 \%(21 / 25)$, respectively, in the intervention group $(P=.39)$. At the end of follow-up, 40\% (4/10) frail and 40\% (4/10) prefrail were found in the control group, compared to $19 \%(3 / 16)$ and $62 \%(10 / 16)$, respectively, in the intervention group $(P=.58)$. Between visit one and visit four, $10 \%(1 / 10)$ improved their frailty status in the control group versus $19 \%(3 / 16)$ in the intervention group. However, these differences were not significant. Concerning the evolution of the physical activity criterion during follow-up, there was no significant differences despite a trend toward a more sedentary lifestyle in both groups. The overall EQ-5D score, SF-36 score, and functional scores (ADL, IADL, and SPPB) did not show any significant difference between the two groups.

Two notable adverse events not attributable to the intervention were reported, one in each group: 1 participant in the control group had a fall and 1 participant in the intervention group had a fracture.

\section{Health Costs, Installation, and Maintenance Costs}

Intend to treat analysis results showed a trend in favor of the intervention group in terms of costs (Table 5).

Table 5. Descriptive cost data.

\begin{tabular}{llll}
\hline Cost data & Control & Intervention & $P$ value \\
\hline Medical visits total costs $\left(€^{\mathrm{a}}\right)$ & 2001.00 & 1051.00 & N/A \\
Hospitalization total cost $(€)$ & $15,374.60$ & 6751.10 & N/A \\
Total costs $(€)$ & $17,375.60$ & 7802.10 & N/A \\
Medical visits, $n$ & 87 & 45 & N/A \\
Hospitalization stays, $\mathrm{n}$ & 11 & 7 & N/A \\
Medical visits cost $(€)$, mean (SD) & $111.20(124.8)$ & $27.70(120.9)$ & .03 \\
Hospitalization cost $(€)$, mean (SD) & $854.10(1245.20)$ & $177.70(1202.80)$ & .21 \\
Total cost $(€)$, mean $(\mathrm{SD})$ & $965.30(1329.90)$ & $205,32(1284.90)$ & .049 \\
\hline
\end{tabular}

${ }^{\mathrm{a}} \mathrm{A}$ currency exchange rate of $€ 1=\mathrm{US} \$ 1.2$ is applicable. 
A commercial company (SADIR Assistance) set up home installation and calibration protocol. They spent on average 33.9 (SD 13.1) minutes per visit per person during the study, for an average number of 3.8 (SD 0.6) visits per person (including the installation visit). The mean cost for installation and maintenance communicated by the company was $€ 47.7$ (SD $€ 9.7$ ) per month per person (a currency exchange rate of $€ 1=\mathrm{US}$ $\$ 1.2$ is applicable).

\section{Discussion}

\section{Principal Results and Limitations}

Our 12-week field trial is the first to assess participant's acceptability of an instrumented insole over 12 weeks in frail older adults in a real-life setting. Difficulty of physical activity follow-up in older individuals and insufficient in-person measures and self-reported data limit clinical research in this domain $[9,22]$. Currently, there is limited data on the use of instrumented insoles beyond a few hours of laboratory testing.

This study confirmed the importance of a multiphase design for health technologies. The living lab participants valued the study's participative aspect and proposed modifications concerning both the software and the hardware, including the manufacturing method of the insole. Our living lab tests introduced an optimized reality between technical lab tests [26] and an ongoing real-life field trial. User feedback provided technical and acceptability issues, which were fixed before the field trial (low reliability scores, mainly associated with synchronization problems between the insole and the touchpad). However, despite these precautions, certain technical problems affected the field trial.

Participants validated the device's design: induction charger, charging routine, battery autonomy, and data transfer automation. The insole calibration phase raised no user concern. These are important results as the device was specifically designed to ensure its unobtrusiveness. There were several reasons to choose an insole: it is unobtrusive and can be worn without disturbing or stigmatizing the person, several studies showed that inertial sensors worn on the feet allow accurate walking speed measurement $[27,43,44]$, and the user does not need to remember wearing it.

Semistructured interviews and questionnaires at the end of follow-up reported that the insole was actually worn, unobtrusive, and well tolerated. Moreover, participants were compliant during the 3-month follow-up; this was confirmed by objective data measured by the insole. Most participants claimed that the insole was comfortable and did not cause any discomfort. This result was innovative, as it is one of the first to describe insole long-term wearing acceptability in older adults who are frail. Thus, a thin instrumentation including a flexible battery was designed. This technical challenge remains and must be considered when instrumenting an insole because it is one of the main participant complaints. Acceptability of wearable devices is the cornerstone of large implementation in real-life settings. Finally, those who did not walk regularly also expressed the fact that wearing a smart insole could encourage walking, which remains to be proven.
We found no significant differences between groups in terms of physical activity or frailty evolution. This could be explained by the low study power and by the fact that our touchpad did not offer any incentive or educational content. Information and communication technology-supported lifestyle programs and motion sensing-based monitoring can influence daily physical activity thanks to feedback [45]. However, adding educational content is more efficient $[46,47]$.

This study included a small sample of volunteer older participants. Even if a few exclusion criteria were applied, there was probably a recruitment bias due to highly motivated participants. Moreover, a large proportion of participants in the intervention group discontinued the intervention because of technical issues.

The results obtained by this semiqualitative approach are not free of the usual biases, in particular external validity. There are other limitations to the generalizability of our results. Certain participants brought up the difficulty of integrating an insole in their shoes (eg, sandals), which could be even more problematic in countries where people walk barefoot indoor or wear outdoor shoes.

Moreover, the use of the touchpad interface was not satisfying because of lack of technical support for using it and participant's computer literacy, web interface ergonomic issues, and the absence of educational content (eg, video tutorial). These shortcomings led most of the participants not to use the touchpad. The motivational aspect of our device was poor despite the potential interest this could have [48]. In a second development phase, an educational and motivational network should be developed to increase user's adherence. It seems important to also include participant's computer literacy when developing health devices in this population to ensure better acceptability.

Previous work has highlighted the difficulty of implementing such technical devices in health care practice due to the importance of material and human investment [49]. The results of this study give a glimpse into health device development and improvement, but further studies are required in a larger population to improve large-scale implementation.

\section{Comparison With Prior Work}

Previous studies have shown the possibility to monitor mobility-related activities based on motion sensors, but few explicitly mentioned acceptability issues or used experimental research designs to evaluate clinical applications in older people $[16,22,50,51]$. Most systems consisting of multiple sensors or devices are difficult when applied in long-term monitoring in real-life (eg, the DynaPort MoveMonitor weighs $44.5 \mathrm{~g}$ and is fixed with an elastic belt [15]). A few studies evaluated accelerometer-based devices on short treadmill walks [52] on a short time period at home, ranging from a few minutes to days [15,53-56] with up to 20 older participants with unknown frailty status.

Most of these studies explore various aspects of walking such as depth posture and activity detection. Nevertheless, none of these studies evaluated long-term acceptability of such wearable 
devices in monitoring walking activity in an older participant sample.

Kaye and colleagues [57] conducted the most notable study. They evaluated in-home walking monitoring using embedded sensors in autonomous living older individuals during a 4-week period. The study did not specifically target frailty status monitoring and was limited to indoor activity. They were able to accurately monitor mean walking speed and even predict falls [9]. Embedded and wearable tools to monitor walking activity provide complementary information. Outdoor walking speed analysis has advantages on indoor measurements because walking distance may be longer and informs global aerobic physical activity [22,58].

Consumer-grade activity trackers (connected devices or smartphone apps) are increasingly popular. However, there is a lack of real-life research on the device's performance in older adults. Given the inaccuracy of these applications, caution is required in promoting self-monitoring physical activity and their use for health prediction $[19,23,24]$. Indeed, the absence of step length calibration does not allow accurate measures of walking speed and walking characterization. Another major concern is their lack of acceptability in frail older adults. Most of them require minimal computer literacy, and users have to remember to wear it every day. Moreover, there is many constraints related to obtrusiveness (eg, device charging and data transfer). Lastly, the algorithms used to measure steps and other metrics are typically proprietary and may not be available to investigators [59].

\section{Conclusion and Perspectives}

Wearable connected sensors are promising for real-life monitoring and appear to be a solution in improving physical activity promotion in frail older adults. However, optimal deployment of wearable health devices will require further research conducted in real-life conditions to test acceptability, effectiveness, and costs. This study reports real-life acceptability data on an instrumented insole in frail older participants over a 12-week period. These results are informative in terms of technical choices for those who wish to instrument a shoe insole.

This field of research is essential and offers interesting perspectives. Along physical activity promotion, these tools would improve detection of early preclinical health transitions implicated in decreased physical performance $[9,57,60,61]$ (eg, gait speed variability over time). Thus, continuous measurements would also enable identification of innovative "digital biomarkers" as a complementary solution to "traditional" biomarkers, leading to more personalized interventions.

\section{Acknowledgments}

This study was funded by the French National Research Agency and National Health and Autonomy Funding Agency through the TECSAN program (Project Number ANR-13-TECS-0007-2013-RESPECT). The funder does not have any roles in the design of this study, execution of the trial, analysis and interpretation of the trial data, and research publications.

\section{Conflicts of Interest}

None declared.

\section{References}

1. Fried LP, Tangen CM, Walston J, Newman AB, Hirsch C, Gottdiener J, Cardiovascular Health Study Collaborative Research Group. Frailty in older adults: evidence for a phenotype. J Gerontol A Biol Sci Med Sci 2001 Mar;56(3):M146-M156. [doi: 10.1093/gerona/56.3.m146] [Medline: 11253156]

2. Metzelthin SF, van Rossum E, de Witte LP, Ambergen AW, Hobma SO, Sipers W, et al. Effectiveness of interdisciplinary primary care approach to reduce disability in community dwelling frail older people: cluster randomised controlled trial. BMJ 2013 Sep 10;347:f5264 [FREE Full text] [doi: 10.1136/bmj.f5264] [Medline: 24022033]

3. Pahor M, Guralnik JM, Ambrosius WT, Blair S, Bonds DE, Church TS, LIFE study investigators. Effect of structured physical activity on prevention of major mobility disability in older adults: the LIFE study randomized clinical trial. JAMA 2014 Jun 18;311(23):2387-2396 [FREE Full text] [doi: 10.1001/jama.2014.5616] [Medline: 24866862]

4. Dedeyne L, Deschodt M, Verschueren S, Tournoy J, Gielen E. Effects of multi-domain interventions in (pre)frail elderly on frailty, functional, and cognitive status: a systematic review. Clin Interv Aging 2017;12:873-896. [doi: 10.2147/CIA.S130794] [Medline: 28579766]

5. Beard JR, Officer A, de Carvalho IA, Sadana R, Pot AM, Michel J, et al. The World report on ageing and health: a policy framework for healthy ageing. Lancet 2016 May 21;387(10033):2145-2154 [FREE Full text] [doi: 10.1016/S0140-6736(15)00516-4] [Medline: 26520231]

6. Cesari M, Araujo de Carvalho I, Amuthavalli Thiyagarajan J, Cooper C, Martin FC, Reginster J, et al. Evidence for the domains supporting the construct of intrinsic capacity. J Gerontol A Biol Sci Med Sci 2018 Nov 10;73(12):1653-1660. [doi: 10.1093/gerona/gly011] [Medline: 29408961]

7. Cesari M, Kritchevsky SB, Penninx BWHJ, Nicklas BJ, Simonsick EM, Newman AB, et al. Prognostic value of usual gait speed in well-functioning older people--results from the Health, Aging and Body Composition Study. J Am Geriatr Soc 2005 Oct;53(10):1675-1680. [doi: 10.1111/j.1532-5415.2005.53501.x] [Medline: 16181165] 
8. Purser JL, Kuchibhatla MN, Fillenbaum GG, Harding T, Peterson ED, Alexander KP. Identifying frailty in hospitalized older adults with significant coronary artery disease. J Am Geriatr Soc 2006 Nov;54(11):1674-1681. [doi: 10.1111/j.1532-5415.2006.00914.x] [Medline: 17087693 ]

9. Piau A, Mattek N, Crissey R, Beattie Z, Dodge H, Kaye J. When will my patient fall? Sensor-based in-home walking speed identifies future falls in older adults. J Gerontol A Biol Sci Med Sci 2020 Apr 17;75(5):968-973 [FREE Full text] [doi: 10.1093/gerona/glz128] [Medline: 31095283]

10. Wild KV, Mattek N, Austin D, Kaye JA. "Are You Sure?": lapses in self-reported activities among healthy older adults reporting online. J Appl Gerontol 2016 Jun;35(6):627-641 [FREE Full text] [doi: 10.1177/0733464815570667] [Medline: 25669877]

11. Liu S, Eaton CB, Driban JB, McAlindon TE, Lapane KL. Comparison of self-report and objective measures of physical activity in US adults with osteoarthritis. Rheumatol Int 2016 Oct;36(10):1355-1364. [doi: 10.1007/s00296-016-3537-9] [Medline: 27435920]

12. Lyons BE, Austin D, Seelye A, Petersen J, Yeargers J, Riley T, et al. Pervasive computing technologies to continuously assess Alzheimer's disease progression and intervention efficacy. Front Aging Neurosci 2015;7:102. [doi: 10.3389/fnagi.2015.00102] [Medline: 26113819]

13. Kaye JA, Maxwell SA, Mattek N, Hayes TL, Dodge H, Pavel M, et al. Intelligent systems for assessing aging changes: home-based, unobtrusive, and continuous assessment of aging. J Gerontol B Psychol Sci Soc Sci 2011 Jul;66 Suppl 1:i180-i190 [FREE Full text] [doi: 10.1093/geronb/gbq095] [Medline: 21743050]

14. Seelye A, Mattek N, Sharma N, Riley T, Austin J, Wild K, et al. Weekly observations of online survey metadata obtained through home computer use allow for detection of changes in everyday cognition before transition to mild cognitive impairment. Alzheimers Dement 2018 Feb;14(2):187-194 [FREE Full text] [doi: 10.1016/j.jalz.2017.07.756] [Medline: 29107052]

15. Taylor LM, Klenk J, Maney AJ, Kerse N, Macdonald BM, Maddison R. Validation of a body-worn accelerometer to measure activity patterns in octogenarians. Arch Phys Med Rehabil 2014 May;95(5):930-934. [doi: 10.1016/j.apmr.2014.01.013] [Medline: 24486241]

16. de Bruin ED, Hartmann A, Uebelhart D, Murer K, Zijlstra W. Wearable systems for monitoring mobility-related activities in older people: a systematic review. Clin Rehabil 2008;22(10-11):878-895. [doi: 10.1177/0269215508090675] [Medline: 18955420]

17. Kramer J, Kowatsch T. Using feedback to promote physical activity: the role of the feedback sign. J Med Internet Res 2017 Jun 02;19(6):e192 [FREE Full text] [doi: 10.2196/jmir.7012] [Medline: 28576757]

18. Geraedts H, Zijlstra A, Bulstra SK, Stevens M, Zijlstra W. Effects of remote feedback in home-based physical activity interventions for older adults: a systematic review. Patient Educ Couns 2013 Apr;91(1):14-24. [doi: 10.1016/j.pec.2012.10.018] [Medline: 23194823]

19. Orr K, Howe HS, Omran J, Smith KA, Palmateer TM, Ma AE, et al. Validity of smartphone pedometer applications. BMC Res Notes 2015 Nov 30;8:733 [FREE Full text] [doi: 10.1186/s13104-015-1705-8] [Medline: 26621351]

20. Toosizadeh N, Joseph B, Heusser MR, Orouji Jokar T, Mohler J, Phelan HA, et al. Assessing upper-extremity motion: an innovative, objective method to identify frailty in older bed-bound trauma patients. J Am Coll Surg 2016 Aug;223(2):240-248 [FREE Full text] [doi: 10.1016/j.jamcollsurg.2016.03.030] [Medline: 27155751]

21. Piau A, Wild K. Performance of e-health devices for frailty evaluation in real-life settings is far from being demonstrated. Gerontology 2019;65(3):309-310 [FREE Full text] [doi: 10.1159/000495208] [Medline: 30783068]

22. Schwenk M, Howe C, Saleh A, Mohler J, Grewal G, Armstrong D, et al. Frailty and technology: a systematic review of gait analysis in those with frailty. Gerontology 2014;60(1):79-89 [FREE Full text] [doi: 10.1159/000354211] [Medline: 23949441]

23. Gomersall SR, Ng N, Burton NW, Pavey TG, Gilson ND, Brown WJ. Estimating physical activity and sedentary behavior in a free-living context: a pragmatic comparison of consumer-based activity trackers and ActiGraph accelerometry. J Med Internet Res 2016 Sep 07;18(9):e239 [FREE Full text] [doi: 10.2196/jmir.5531] [Medline: 27604226]

24. Block VAJ, Pitsch E, Tahir P, Cree BAC, Allen DD, Gelfand JM. Remote physical activity monitoring in neurological disease: a systematic review. PLoS One 2016;11(4):e0154335 [FREE Full text] [doi: 10.1371/journal.pone.0154335] [Medline: 27124611]

25. Ngueleu AM, Blanchette AK, Maltais D, Moffet H, McFadyen BJ, Bouyer L, et al. Validity of instrumented insoles for step counting, posture and activity recognition: a systematic review. Sensors (Basel) 2019 May 28;19(11):2438 [FREE Full text] [doi: 10.3390/s19112438] [Medline: 31141973]

26. Piau A, Charlon Y, Campo E, Vellas B, Nourhashemi F. A smart insole to promote healthy aging for frail elderly individuals: specifications, design, and preliminary results. JMIR Rehabil Assist Technol 2015 May 25;2(1):e5 [FREE Full text] [doi: 10.2196/rehab.4084] [Medline: 28582238]

27. Mariani B, Hoskovec C, Rochat S, Büla C, Penders J, Aminian K. 3D gait assessment in young and elderly subjects using foot-worn inertial sensors. J Biomech 2010 Nov 16;43(15):2999-3006. [doi: 10.1016/j.jbiomech.2010.07.003] [Medline: 20656291] 
28. Campbell NC, Murray E, Darbyshire J, Emery J, Farmer A, Griffiths F, et al. Designing and evaluating complex interventions to improve health care. BMJ 2007 Mar 03;334(7591):455-459 [FREE Full text] [doi: 10.1136/bmj.39108.379965.BE] [Medline: 17332585$]$

29. Sorenson C, Drummond M, Borlum KF, Busse R. How can the impact of health technology assessments be enhanced? Policy Brief - WHO European Ministerial Conference on Health Systems. 2008 Feb Presented at: Health Systems and Policy Analysis; February 2008; Geneva.

30. Chilcott J, Tappenden P, Rawdin A, Johnson M, Kaltenthaler E, Paisley S, et al. Avoiding and identifying errors in health technology assessment models: qualitative study and methodological review. Health Technol Assess 2010 May;14(25):iii-iv, ix. [doi: 10.3310/hta14250] [Medline: 20501062]

31. Arain M, Campbell MJ, Cooper CL, Lancaster GA. What is a pilot or feasibility study? A review of current practice and editorial policy. BMC Med Res Methodol 2010 Jul 16;10:67 [FREE Full text] [doi: 10.1186/1471-2288-10-67] [Medline: 20637084]

32. Sparling PB, Howard BJ, Dunstan DW, Owen N. Recommendations for physical activity in older adults. BMJ 2015 Jan 21;350:h100. [doi: 10.1136/bmj.h100] [Medline: 25608694]

33. Katz S, Ford A, Moskiwitz R. Studies of illness in the aged. The index of ADL: a standardized measure of biological and psychosocial function. JAMA 1963 Sep 21;185:914-919. [Medline: 14044222]

34. Folstein MF, Folstein SE, McHugh PR. "Mini-mental state". A practical method for grading the cognitive state of patients for the clinician. J Psychiatr Res 1975 Nov;12(3):189-198. [doi: 10.1016/0022-3956(75)90026-6] [Medline: 1202204]

35. Lawton MP, Brody EM. Assessment of older people: self-maintaining and instrumental activities of daily living. Gerontologist 1969;9(3):179-186. [Medline: 5349366]

36. Guralnik JM, Ferrucci L, Simonsick EM, Salive ME, Wallace RB. Lower-extremity function in persons over the age of 70 years as a predictor of subsequent disability. N Engl J Med 1995 Mar 02;332(9):556-561. [doi:

10.1056/NEJM199503023320902] [Medline: 7838189]

37. Demers L, Monette M, Lapierre Y, Arnold DL, Wolfson C. Reliability, validity, and applicability of the Quebec User Evaluation of Satisfaction with assistive Technology (QUEST 2.0) for adults with multiple sclerosis. Disabil Rehabil 2002;24(1-3):21-30. [doi: 10.1080/09638280110066352] [Medline: 11827151]

38. Ware JE, Gandek B. Overview of the SF-36 Health Survey and the International Quality of Life Assessment (IQOLA) Project. J Clin Epidemiol 1998 Nov;51(11):903-912. [doi: 10.1016/s0895-4356(98)00081-x] [Medline: 9817107]

39. Dolan P. Modeling valuations for EuroQol health states. Med Care 1997 Nov;35(11):1095-1108. [doi: 10.1097/00005650-199711000-00002] [Medline: 9366889]

40. Hsieh H, Shannon SE. Three approaches to qualitative content analysis. Qual Health Res 2005 Nov;15(9):1277-1288. [doi: 10.1177/1049732305276687] [Medline: 16204405]

41. Lancaster GA, Dodd S, Williamson PR. Design and analysis of pilot studies: recommendations for good practice. J Eval Clin Pract 2004 May;10(2):307-312. [doi: 10.1111/j..2002.384.doc.x] [Medline: 15189396]

42. Eysenbach G, CONSORT-EHEALTH Group. CONSORT-EHEALTH: improving and standardizing evaluation reports of Web-based and mobile health interventions. J Med Internet Res 2011 Dec 31;13(4):e126 [FREE Full text] [doi: 10.2196/jmir.1923] [Medline: 22209829]

43. Martin Schepers H, van Asseldonk EHF, Baten CTM, Veltink PH. Ambulatory estimation of foot placement during walking using inertial sensors. J Biomech 2010 Dec 01;43(16):3138-3143. [doi: 10.1016/j.jbiomech.2010.07.039] [Medline: 20723901]

44. Tien I, Glaser SD, Bajcsy R, Goodin DS, Aminoff MJ. Results of using a wireless inertial measuring system to quantify gait motions in control subjects. IEEE Trans Inf Technol Biomed 2010 Jul;14(4):904-915. [doi: 10.1109/TITB.2009.2021650] [Medline: 19423449]

45. Chow CK, Redfern J, Hillis GS, Thakkar J, Santo K, Hackett ML, et al. Effect of lifestyle-focused text messaging on risk factor modification in patients with coronary heart disease: a randomized clinical trial. JAMA 2015;314(12):1255-1263. [doi: 10.1001/jama.2015.10945] [Medline: 26393848]

46. Bickmore TW, Silliman RA, Nelson K, Cheng DM, Winter M, Henault L, et al. A randomized controlled trial of an automated exercise coach for older adults. J Am Geriatr Soc 2013 Oct;61(10):1676-1683. [doi: 10.1111/jgs. 12449] [Medline: 24001030]

47. van het Reve E, Silveira P, Daniel F, Casati F, de Bruin ED. Tablet-based strength-balance training to motivate and improve adherence to exercise in independently living older people: part 2 of a phase II preclinical exploratory trial. J Med Internet Res 2014 Jun 25;16(6):e159 [FREE Full text] [doi: 10.2196/jmir.3055] [Medline: 24966165]

48. Kwan RY, Lee D, Lee PH, Tse M, Cheung DS, Thiamwong L, et al. Effects of an mHealth brisk walking intervention on increasing physical activity in older people with cognitive frailty: pilot randomized controlled trial. JMIR Mhealth Uhealth 2020 Jul 31;8(7):e16596 [FREE Full text] [doi: 10.2196/16596] [Medline: 32735218]

49. Adlam T, Ulrich E, Kent M, Malinzak L. Frailty testing pilot study: pros and pitfalls. J Clin Med Res 2018 Feb;10(2):82-87 [FREE Full text] [doi: 10.14740/jocmr3203w] [Medline: 29317953] 
50. Gilgen-Ammann R, Schweizer T, Wyss T. Accuracy of distance recordings in eight positioning-enabled sport watches: instrument validation study. JMIR Mhealth Uhealth 2020 Jun 24;8(6):e17118 [FREE Full text] [doi: 10.2196/17118] [Medline: $\underline{32396865]}$

51. Katzan I, Schuster A, Kinzy T. Physical activity monitoring using a Fitbit device in ischemic stroke patients: prospective cohort feasibility study. JMIR Mhealth Uhealth 2021 Jan 19;9(1):e14494 [FREE Full text] [doi: 10.2196/14494] [Medline: 33464213]

52. Huijben B, van Schooten K, van Dieën JH, Pijnappels M. The effect of walking speed on quality of gait in older adults. Gait Posture 2018 Sep;65:112-116. [doi: 10.1016/j.gaitpost.2018.07.004] [Medline: 30558916]

53. Dijkstra B, Kamsma Y, Zijlstra W. Detection of gait and postures using a miniaturised triaxial accelerometer-based system: accuracy in community-dwelling older adults. Age Ageing 2010 Mar;39(2):259-262. [doi: 10.1093/ageing/afp249] [Medline: $\underline{20083616}$ ]

54. Hollewand AM, Spijkerman AG, Bilo HJG, Kleefstra N, Kamsma Y, van Hateren KJJ. Validity of an accelerometer-based activity monitor system for measuring physical activity in frail older adults. J Aging Phys Act 2016 Oct;24(4):555-558. [doi: 10.1123/japa.2014-0290] [Medline: 26964560]

55. Culhane KM, Lyons GM, Hilton D, Grace PA, Lyons D. Long-term mobility monitoring of older adults using accelerometers in a clinical environment. Clin Rehabil 2004 May;18(3):335-343. [doi: 10.1191/0269215504cr734oa] [Medline: 15137565]

56. Kim B, McKay SM, Lee J. Consumer-grade wearable device for predicting frailty in Canadian home care service clients: prospective Observational proof-of-concept study. J Med Internet Res 2020 Sep 03;22(9):e19732 [FREE Full text] [doi: 10.2196/19732] [Medline: 32880582]

57. Kaye J, Mattek N, Dodge H, Buracchio T, Austin D, Hagler S, et al. One walk a year to 1000 within a year: continuous in-home unobtrusive gait assessment of older adults. Gait Posture 2012 Feb;35(2):197-202 [FREE Full text] [doi: 10.1016/j.gaitpost.2011.09.006] [Medline: 22047773]

58. Najafi B, Khan T, Wrobel J. Laboratory in a box: wearable sensors and its advantages for gait analysis. Annu Int Conf IEEE Eng Med Biol Soc 2011;2011:6507-6510. [doi: 10.1109/IEMBS.2011.6091605] [Medline: 22255829]

59. Seo M, Shin M, Park TS, Park J. Clinometric gait analysis using smart insoles in patients with hemiplegia after stroke: pilot study. JMIR Mhealth Uhealth 2020 Sep 10;8(9):e22208 [FREE Full text] [doi: 10.2196/22208] [Medline: 32909949]

60. Dodge HH, Zhu J, Harvey D, Saito N, Silbert LC, Kaye JA, Alzheimer's Disease Neuroimaging Initiative. Biomarker progressions explain higher variability in stage-specific cognitive decline than baseline values in Alzheimer disease. Alzheimers Dement 2014 Nov;10(6):690-703 [FREE Full text] [doi: 10.1016/j.jalz.2014.04.513] [Medline: 25022534]

61. Piau A, Wild K, Mattek N, Kaye J. Current state of digital biomarker technologies for real-life, home-based monitoring of cognitive Function for Mild Cognitive Impairment to Mild Alzheimer Disease and Implications for clinical care: systematic review. J Med Internet Res 2019 Aug 30;21(8):e12785 [FREE Full text] [doi: 10.2196/12785] [Medline: 31471958]

\author{
Abbreviations \\ ADL: Activity of Daily Living \\ CONSORT: Consolidated Standards of Reporting Trials \\ GHF: geriatric day hospital for frailty \\ IADL: Instrumental Activities of Daily Living \\ MMSE: Mini-Mental State Examination \\ SF-36: $36-$ Item Short Form Health Survey \\ SPPB: Short Physical Performance Battery
}

Edited by L Buis; submitted 25.07.19; peer-reviewed by M Yuhas, A Gros, P Robert, H Verloo, E Sadeghi-Demneh, D López López;
comments to author 06.09.20; revised version received 02.02.21; accepted 14.04.21; published 05.07.21
Please cite as:
Piau A, Steinmeyer Z, Charlon Y, Courbet L, Rialle V, Lepage B, Campo E, Nourhashemi F
A Smart Shoe Insole to Monitor Frail Older Adults'Walking Speed: Results of Two Evaluation Phases Completed in a Living Lab
and Through a 12-Week Pilot Study
JMIR Mhealth Uhealth 2021;9(7):e15641
URL: https://mhealth.jmir.org/2021/7/e15641
doi: $\underline{10.2196 / 15641}$
PMID:

(C)Antoine Piau, Zara Steinmeyer, Yoann Charlon, Laetitia Courbet, Vincent Rialle, Benoit Lepage, Eric Campo, Fati Nourhashemi. Originally published in JMIR mHealth and uHealth (https://mhealth.jmir.org), 05.07.2021. This is an open-access article distributed 
under the terms of the Creative Commons Attribution License (https://creativecommons.org/licenses/by/4.0/), which permits unrestricted use, distribution, and reproduction in any medium, provided the original work, first published in JMIR mHealth and uHealth, is properly cited. The complete bibliographic information, a link to the original publication on https://mhealth.jmir.org/, as well as this copyright and license information must be included. 